Linköping studies in

science and technology. Dissertations

(2015): nr 1714

\title{
Carrier Lifetime Relevant Deep Levels In $\mathrm{SiC}$
}

\author{
Ian D. Booker
}

Linköping University Electronic Press

Linköping, Sweden

http://urn.kb.se/resolve?urn=urn:nbn:se:liu:diva-121515 
Published on September 12, 2015 by

Linköping University Electronic Press

58183 Linköping, Sweden

\author{
Linköping studies in \\ science and technology. Dissertations \\ ISSN 0345-7524 \\ ISBN: 978-91-7685-919-3
}

(C)2015 Ian Don Booker

Typeset by the author using ${ }^{A} T_{E} X$

Formatted using étendu style

\title{
Recommended citation:
}

<Booker, I. D.>. < Carrier Lifetime Relevant Deep Levels in SiC>. Linköping studies in science and technology. Dissertations, (2015): $n$ r 1714. http://

www.ep.liu.se/ea/cis/2015/1714/. September 12, 2015.

This URL will also contain a link to the author's home page.

The publishers will keep this article on-line on the Internet

(or its possible replacement network in the future)

for a period of 25 years from the date of publication,

barring exceptional circumstances as described separately.

The on-line availability of the article implies

a permanent permission for anyone to read the article on-line,

to print out single copies of it, and to use it unchanged

for any non-commercial research and educational purpose,

including making copies for classroom use.

This permission can not be revoked by subsequent transfers of copyright. All other uses of the article are conditional on the consent of the copyright owner.

The publication of the article on the date stated above included also the production of a limited number of copies on paper, which were archived in Swedish university libraries like all other written works published in Sweden.

The publisher has taken technical and administrative measures to assure that the on-line version of the article will be permanently accessible using the URL stated above, unchanged, and permanently equal to the archived printed copies at least until the expiration of the publication period.

For additional information about the Linköping University Electronic Press and its procedures for publication and for assurance of document integrity, please refer to its $W W W$ home page: http://www.ep.liu.se/ or by conventional mail to the address stated above. 


\begin{abstract}
Silicon carbide ( $\mathrm{SiC}$ ) is currently under development for high power bipolar devices such as insulated gate bipolar transistors (IGBTs). A major issue for these devices is the charge carrier lifetime, which, in the absence of structural defects such as dislocations, is influenced by point defects and their associated deep levels. These defects provide energy levels within the bandgap and may act as either recombination or trapping centers, depending on whether they interact with both conduction and valence band or only one of the two bands. Of all deep levels know in $4 \mathrm{H}-$ $\mathrm{SiC}$, the intrinsic carbon vacancy related $\mathrm{Z}_{1 / 2}$ is the most problematic since it is a very effective recombination center which is unavoidably formed during growth. Its concentration in the epilayer can be decreased for the production of high voltage devices by injecting interstitial carbon, for example by oxidation, which, however, results in the formation of other new deep levels. Apart from intrinsic crystal flaws, extrinsic defects such as transition metals may also produce deep levels within the bandgap, which in literature have so far only been shown to produce trapping effects.
\end{abstract}

The focus of the thesis is the transient electrical and optical characterization of deep levels in $\mathrm{SiC}$ and their influence on the carrier lifetime. For this purpose, deep level transient spectroscopy (DLTS) and minority carrier transient spectroscopy (MCTS) variations were used in combination with time-resolved photoluminescence (TRPL). Paper 1 deals with a lifetime limiting deep level related to Fe-incorporation in n-type $4 \mathrm{H}-\mathrm{SiC}$ during growth and papers 2 and 3 focus on identifying the main intrinsic recombination center in p-type $4 \mathrm{H}-\mathrm{SiC}$. In paper 4 , the details of the charge carrier capture behavior of the deeper donor levels of the carbon vacancy, $\mathrm{EH}_{6 / 7}$, are investigated. Paper 5 deals with trapping effects created by unwanted incorporation of high amounts of boron during growth of n-type $4 \mathrm{H}-\mathrm{SiC}$ which hinders the measurement of the carrier lifetime by room temperature TRPL. Finally, paper 6 is concerned with the characterization of oxidation-induced deep levels created in n- and p-type $4 \mathrm{H}$ - and $6 \mathrm{H}-\mathrm{SiC}$ as a side-product of lifetime improvement by oxidation. 
In paper 1 , the appearance of a new recombination center in n-type $4 \mathrm{H}-\mathrm{SiC}$, the RB1 level is discussed and the material is analyzed using room temperature TRPL, DLTS and pn-junction DLTS. The level appears to originate from a reactor contamination with $\mathrm{Fe}$, a transition metal that generally leads to the formation of several trapping centers in the bandgap. Here it is found that under specific circumstances beneficial to the growth of high-quality material with a low $\mathrm{Z}_{1 / 2}$ concentration, the Fe incorporation also creates an additional recombination center capable of limiting the carrier lifetime.

In paper 2, all deep levels found in p-type 4H-SiC grown at Linköping University which are accessible by DLTS and MCTS are investigated with regard to their efficiency as recombination centers. We find that none of the detectable levels is able to reduce carrier lifetime in p-type significantly, which points to the lifetime killer being located in the top half of the bandgap and having a large hole to electron capture cross section ratio (such as $\mathrm{Z}_{1 / 2}$ in n-type material), making it undetectable by DLTS and MCTS. Paper 3 compares carrier lifetimes measured by temperature-dependent TRPL measurements in n- and ptype $4 \mathrm{H}-\mathrm{SiC}$ and it is shown that the lifetime development over a large temperature range $(77-1000 \mathrm{~K})$ is similar in both types. This is interpreted as a further indication that the carbon vacancy related $\mathrm{Z}_{1 / 2}$ level is the main lifetime killer in p-type.

In paper 4 , the hole and electron capture cross sections of the near midgap deep levels $\mathrm{EH}_{6 / 7}$ are characterized. Both levels are capable of rapid electron capture but have only small hole capture rates, making them insignificant as recombination centers, despite their advantageous position near midgap.

Minority carrier trapping by boron, which is both a p-type dopant and an unavoidable contaminant in $4 \mathrm{H}-\mathrm{SiC}$ grown by CVD, is investigated in paper 5. Since even the shallow boron acceptor levels are relatively deep in the bandgap, minority trapand-release effects are detectable in room-temperature TRPL measurements. In case a high density of boron exists in n-type $4 \mathrm{H}-\mathrm{SiC}$, for example leached out from damaged graphite reactor parts during growth, we demonstrate that these trapping 
effects may be misinterpreted in room temperature TRPL measurements as a long free carrier lifetime.

Paper 6 uses MCTS, DLTS, and room temperature TRPL to characterize the oxidation induced deep levels ON1 and ON2 in n- and p-type 4H- and their counterparts OS1-OS3 in 6H$\mathrm{SiC}$. The levels are found to all be positive-U, coupled two-levels defects which trap electrons efficiently but exhibit very inefficient hole capture once the defect is fully occupied by electrons. It is shown that these levels are incapable of significantly influencing carrier lifetime in epilayers which underwent high temperature lifetime enhancement oxidations. Due to their high density after oxidation and their high thermal stability they may, however, act to compensate n-type doping in low-doped material.

\section{Authors' Affiliations}

Ian D. Booker

Department of Physics, Chemistry and Biology

Linköping University

SE-58183 Linköping, Sweden

E-mail: $\{$ ianbo\}@ifm.liu.se

Webpage: http://www.ifm.liu.se/

\section{Acknowledgement}

I would like to thank the following people for making this dissertation possible:

- Prof. Einar Ö. Sveinbjörnsson, my main supervisor, for your great support and the countless emails-conversations.

- Prof. Erik Janzén, my second supervisor, for giving me the chance to focus on a number of interesting topics and for your great support.

- Asst. Prof. Jawad ul-Hassan, for your epilayers and discussions.

- Prof. Nguyen Tien Son and Assoc. Prof. Ivan Ivanov for the discussions on deep levels.

- Sven, Roger, Rickard, Ildiko and Eva for technical and administrative support.

- All the others in the semiconductor materials group (Chams, Ted, Martin, Valdas, Robin, Xun, Louise, Björn, Pontus, Pitsiri, Duc, Thang, Milan, Daniel, Jianwu, ...) for some interesting times. 
$\begin{array}{ll}\text { Table of Contents } & 1\end{array}$

1 Motivation 5

$\begin{array}{lll}2 & \text { Silicon carbide } & 7\end{array}$

3 Point defects $\quad 10$

3.1 Shallow and deep levels in the bandgap . . . . . . . . . . 11

3.2 Capture, emission and rate equations for a single level trap . 12

3.3 Capture and recombination processes . . . . . . . . . . 15

3.4 Emissions from deep levels . . . . . . . . . . . . . . . . . 18

4 Schottky diodes and space charge regions 20

5 Experimental methods $\quad 22$

5.1 Capacitance transient measurements

(DLTS, MCTS, O-DLTS, EO-DLTS and OE-MCTS) . . . . . 22

5.2 Multi-spectrum fitting . . . . . . . . . . . . . 23

5.3 Time resolved photoluminescence (TRPL) . . . . . . . . 24

6 Summary of the papers $\quad 26$ 
INCLUDED PAPERS

1. I. D. Booker, J. Hassan, L. Lilja, F. C. Beyer, R. Karhu, J. P. Bergman, Ö. Danielsson, O. Kordina, E. Ö. Sveinbjörnsson and E. Janzén, Carrier Lifetime Controlling Defects $\mathrm{Z}_{1 / 2}$ and RB1 in Standard and Chlorinated Chemistry Grown $4 \mathrm{H}-\mathrm{SiC}$, Cryst. Growth and Design, 14 (6), 4104-4110 (2014).

2. I. D. Booker, J. Hassan, E. Janzén, and E. Ö. Sveinbjörnsson, Electron and hole capture cross sections of deep levels accessible by DLTS and MCTS in $p$-type $4 \mathrm{H}-\mathrm{SiC}$, submitted to J. Appl. Phys.

3. I. D. Booker, J. Hassan, P. Stenberg, E. Janzén, and E. Ö. Sveinbjörnsson, Carrier lifetime in $p$ - and $n$-type $4 \mathrm{H}-\mathrm{SiC}$, manuscript

4. I. D. Booker, J. Hassan, E. Janzén, and E. Ö. Sveinbjörnsson, Donor and double donor transitions of the carbon vacancy related $\mathrm{EH}_{6 / 7}$ deep level in $4 \mathrm{H}-\mathrm{SiC}$, manuscript

5. I. D. Booker, M. Yazdanfar, E. Janzén, and E. Ö. Sveinbjörnsson, The influence of shallow boron and the deep D-center on carrier lifetime in $n$ - and $p$-type $4 \mathrm{H}-\mathrm{SiC}$, submitted to J. Appl. Phys.

6. I. D. Booker, H. Abdalla, J. Hassan, R. Karhu, L. Lilja, E. Janzén, and E. Ö. Sveinbjörnsson, Oxidation induced deep levels in $n$ - and $p$ type $4 \mathrm{H}-$ and $6 \mathrm{H}-\mathrm{SiC}$ and their influence on carrier lifetime, submitted to Phys. Rev. B

\section{My contribution to the papers}

Papers 1-6 I planned the experiments, performed almost all of the DLTS measurements, all of non-DLTS capacitance transient experiments, all TRPL experiments, wrote the manuscript (with some input from the co-authors in paper 1) and finalized the papers. 
1. M. Widmann, S.-Y. Lee, T. Rendler, N. T. Son, H. Fedder, S. Paik, L. P. Yang, N. Zhao, S. Yang, I. Booker, A. Denisenko, M. Jamali, S. A. Momenzadeh, I. Gerhardt, T. Ohshima, A. Gali, E. Janzén, J. Wrachtrup, Nature Materials, 14 (2), 164-168 (2015).

2. I. D. Booker, H. Abdalla, L. Lilja, J. Hassan, J. P. Bergman, E. O. Sveinbjörnsson, and E. Janzén,, Oxidation induced $\mathrm{ON}_{1}, \mathrm{ON}_{2 a / b}$ defects in $4 \mathrm{H}-\mathrm{SiC}$ characterized by DLTS, Mater. Sci. Forum 778-780, 281-284 (2014).

3. B. Kallinger, M. Rommel, L. Lilja, J. ul Hassan, I. Booker, E. Janzén, P. Bergman, Comparison of Carrier Lifetime Measurement and Mapping in 4H-SiC Using Time Resolved Photoluminescence and mu-PCD, Mat. Sci. Forum, 778-780, 301-304 (2014).

4. L. Lilja, I. D. Booker, J. Hassan, E. Janzén, and J. P. Bergman, The influence of growth conditions on carrier lifetime in $4 \mathrm{H}-\mathrm{SiC}$, J. Cryst. Growth, 381, 43-50 (2013).

5. M. Yazdanfar, P. Stenberg, I. D. Booker, I. G. Ivanov, O. Kordina, H. Pedersen, and E. Janzén, Process stability and morphology optimization of very thick $4 \mathrm{H}-\mathrm{SiC}$ epitaxial layers grown by chloride-based CVD, J. Cryst. Growth, 380, 55-60 (2013).

6. J. Hassan, I. D. Booker, L. Lilja, A. Hallén, M. Fagerlind, J. P. Bergman, and E. Janzén, On-axis homoepitaxial growth of $4 \mathrm{H}-\mathrm{SiC}$ PiN structure for high power applications, Mat. Sci. Forum, 740-742, 173-176 (2013).

7. M. Yazdanfar, P. Stenberg, I. D. Booker, I. G. Ivanov, H. Pedersen, O. Kordina, and E. Janzén, Morphology optimization of very thick 4H-SiC epitaxial layers, Mat. Sci. Forum, 740-742, 251-254 (2013).

8. L. Lilja, J. Hassan, I. D. Booker, J. P. Bergman, and E. Janzén, Influence of growth temperature on carrier lifetime in $4 \mathrm{H}-\mathrm{SiC}$ epilayers, Mat. Sci. Forum, 740-742, 637-640 (2013).

9. M. Fagerlind, I. D. Booker, P. Bergman, E. Janzén, H. Zirath, and N. Rorsman, Influence of Large-Aspect-Ratio Surface Roughness on 
Electrical Characteristics of AlGaN/GaN HFETs, IEEE Trans. Device Mater. Rel., 12 (3), 538-546 (2012).

10. J. Hassan, L. Lilja, I. D. Booker, J. P. Bergman, and E. Janzén, Influence of growth mechanism on carrier lifetime in on-axis homoepitaxial layers of 4H-SiC, Mat. Sci. Forum, 717-720, 157-160 (2012).

11. L. Lilja, J. Hassan, I. D. Booker, J. P. Bergman, and E. Janzén, The effect of growth conditions on carrier lifetime in $n$-type $4 \mathrm{H}-\mathrm{SiC}$ epitaxial layers, Mat. Sci. Forum, 717-720, 161-164 (2012).

12. J. P. Bergman, I. D. Booker, L. Lilja, J. Hassan, and E. Janzén, Radial variation of measured carrier lifetimes in epitaxial layers grown with wafer rotation, Mat. Sci. Forum, 717-720, 289-292 (2012).

13. I. D. Booker, J. Hassan, A. Hallén, E. Ö. Sveinbjörnsson, O. Kordina, and J. P. Bergman, Comparison of post-growth lifetime improvement methods for $4 \mathrm{H}-\mathrm{SiC}$ epilayers, Mat. Sci. Forum, 717-720, 285-288 (2012).

14. I. D. Booker, J. Hassan, E. Janzén, and J. P. Bergman, Highresolution time-resolved carrier lifetime and photoluminescence mapping of 4H-SiC epilayers, Mat. Sci. Forum, 717-720, 293-296 (2012). 
After the semiconductor field saw its first initial development with research on germanium $(\mathrm{Ge})$, silicon ( $\mathrm{Si}$ ) quickly became the dominant material as it was relatively easy to produce high quality crystals in large quantities. In the late 1960s, III-V binary materials such as gallium arsenide (GaAs) were introduced for use in microwave and optoelectronic applications. However, for high-temperature, high-power and high-frequency applications, wider bandgap materials such as gallium nitride $(\mathrm{GaN})$ and silicon carbide $(\mathrm{SiC})$ are attractive due to their exceptional material properties such as high electron mobility, high breakdown field, high saturated drift velocity and high thermal conductivity. The latter, $\mathrm{SiC}$ will be the focus of this thesis.

In 1824 J. J. Berzelius synthesizes $\mathrm{SiC}$ for the first time. Larger scale production only followed almost 70 years later, when E. G. Acheson patented a process to manufacture $\mathrm{SiC}$, which he called carborundum, and marketed it as an abrasive material. In 1955 then, J. A. Lely improved Achesonś process. Much of the early research on $\mathrm{SiC}$ was performed on Lely platelets of various polytypes grown by this process, however, this material was still not useful for electronic device applications. One issue complicating mass production of $\mathrm{SiC}$ crystals is the high thermal resistance against stoichiometric melting which makes approaches such as the Czochralski method, which is extensively used in $\mathrm{Si}$, impossible. An important step towards this goal was the development of physical vapor transport (PVT), introduced by Yu. M. Tairov and V. F. Tsvetkov in 1973. The process was further improved at North Carolina State University, where Cree Research Inc. was founded in 1987 and commercialized the research. Additional important steps towards the growth of large, high quality crystals were the developments of vapor phase epitaxy (VPE), chemical vapor deposition (CVD) using a hot-wall reactor and high-temperature CVD (HT-CVD), which combined gas-phase precursors and growth by sublimation.

The most important commercial applications for SiC are its use as substrates for high intensity GaN LEDs and applications in the field of power electronics such as Schottky diodes and transistors for rectifiers and inverters. In 2001, the first commercial Schottky diodes produced by Cree Inc. became available, and today unipolar devices in the 1200-1700 V range are offered by a number of different companies, for example ROHM Co., Ltd., GeneSiC Inc. and Infineon AG. 
While SiC can be used for unipolar devices (Schottky barrier diodes and field effect transistors), its main advantage over competing materials lies in the area of high-voltage bipolar applications (for example bipolar junction transistors) where its material properties make it far superior to its main competitor, Si. An example of a commercial application are the PiN rectifiers and thyristors with up to $13 \mathrm{kV}$ rating currently under development by GeneSiC, Inc..

Despite the maturity $\mathrm{SiC}$ has reached as a semiconductor material, a number of important topics are still under development. Today, research continues for example on growth by chlorinated chemistry, which promises substantially higher growth rates than those achieved by using standard chemistry, or on methods to improve the material carrier lifetime by hightemperature oxidations or carbon implantation followed by annealing.

The aim of this thesis is to contribute to the latter area of research, namely carrier lifetime, its improvement and the characterization and understanding of lifetime limiting defects in $\mathrm{SiC}$. In the following sections, a brief introduction on the crystal structural and the relevant electrical properties of $\mathrm{SiC}$ will be given. Next, electrically active defects, their origin and their influence on charge carriers will be discussed, followed by an overview of the characterization techniques which were used for the research. These are deep level transient spectroscopy (DLTS), optical-DLTS (O-DLTS), minority carrier transient spectroscopy (MCTS), optical-electrical MCTS (OEMCTS) and time-resolved photoluminescence (TRPL). 


\section{CHAPTER 2}

SILICON CARBIDE

The transition from a semiconductor to an insulator is not well defined, and, for many years, while Si and Ge were the dominant semiconducting materials, was set at about a bandgap of $3 \mathrm{eV}$. Most wide bandgap semiconductors however fall near or above this supposed boundary, for example GaN, with its bandgap of $\mathrm{E}_{g}=3.4 \mathrm{eV}, \mathrm{ZnS}\left(\mathrm{E}_{g}=3.6 \mathrm{eV}\right)$, or diamond $\left(\mathrm{E}_{g}=5.5 \mathrm{eV}\right)$ and $\mathrm{AlN}\left(\mathrm{E}_{g}=6.2 \mathrm{eV}\right)$ with even larger bandgaps. $\mathrm{SiC}$, the topic of this thesis, is also one of these wide bandgap semiconductors with an indirect bandgap ranging from $\mathrm{E}_{g}=2.3$ to $3.2 \mathrm{eV}$ varying as a function of its crystal stacking sequence.

Chemically, the $\mathrm{SiC}$ crystal is constructed of an equal amount of $\mathrm{Si}$ and $\mathrm{C}$ atoms. The binary Si-C bonds are nearly covalent, with a small ionic contribution of about $12 \%$, due to Si being slightly more positive and $\mathrm{C}$ being slightly more negative relative to each other. ${ }^{[1]}$ The smallest component of the $\mathrm{SiC}$ crystal lattice is a tetrahedron (Fig. 2.1) of $\mathrm{Si}$ (or C) surrounded by four $\mathrm{C}$ (or $\mathrm{Si}$ ) atoms, with very short, strong $\mathrm{sp}^{3}$ bonds of $0.189 \mathrm{~nm}$ length between the atoms. ${ }^{[2]}$ For orientation purposes the positive $\vec{c}$-axis by definition is the direction in which $\mathrm{Si}$ atoms are found situated directly above $\mathrm{C}$ atoms. By closely packing these tetrahedra, a larger crystal is then constructed which can also be described as being constructed of Si-C bi-layers stacked in a specific sequence. In total three possible ways of arranging the tetrahedra, and thus the stacking sites for atoms above each bi-layer, exist and these are referred to as A, B or C stacking sites (Fig. 2.2). Crystallographically, a stacking sequence of $\mathrm{ABC}$ produces a zincblende structure, while a stacking sequence of only $\mathrm{AB}$ produces a wurtzite structure. An important property of $\mathrm{SiC}$ is its polytypism, which is a 1 dimensional type of polymorphism, where each polytype of $\mathrm{SiC}$ is characterized by its unique stacking sequence. The three most important polytypes are

3C-SiC, the only purely cubic polytype, stacked in an $\mathrm{ABC}$ sequence, $4 \mathrm{H}-\mathrm{SiC}$, with a hexagonal crystal structure stacked in an ABCB sequence, and

$6 \mathrm{H}-\mathrm{SiC}$, another hexagonal polytype with a stacking sequence of $\mathrm{AB}-$ CACB.

The notations used above are referred to as Ramsdell notation, where the letter refers to the symmetry of the crystal ( $\mathrm{C}$ for cubic, $\mathrm{H}$ for hexagonal 


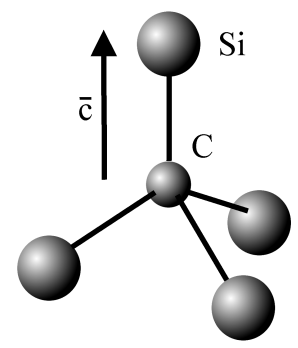

a)

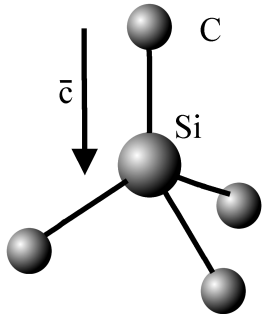

b)

Figure 2.1: A tetrahedron building block of $\mathrm{SiC}$ containing either $\mathrm{C}$ (a) or $\mathrm{Si}(\mathrm{b})$ as the center species.

and $\mathrm{R}$ for rhombohedral) and the number denotes the total count of double layers in one period. The polytypism of $\mathrm{SiC}$ appears to mainly originate from the small differences in the total energies of the different polytypes, which also allows the crystal to easily form stacking faults. ${ }^{[3]}$

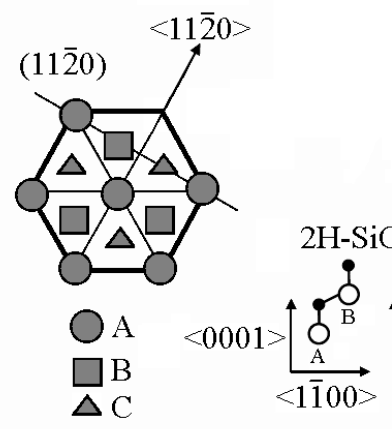

(a)

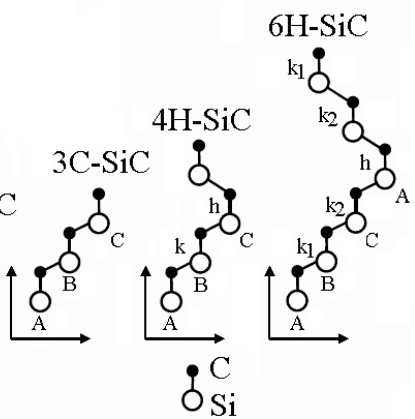

(b)

Figure 2.2: The three possible stacking orders A, B and C (a) and different polytypes with their stacking orders (b).

As a result of the different stacking sequences, material properties and parameters for the polytypes differ strongly. A good example are the lattice constants and bandgaps in the non-cubic polytypes. While the $\vec{a}$-lattice constant is $0.308 \mathrm{~nm}$ for $2 \mathrm{H}, 4 \mathrm{H}$ - and $6 \mathrm{H}-\mathrm{SiC}$, the $\vec{c}$-lattice constant increases from $0.505 \mathrm{~nm}$ in $2 \mathrm{H}-\mathrm{SiC}$ to $1.008 \mathrm{~nm}$ in $4 \mathrm{H}-\mathrm{SiC}$ to $1.512 \mathrm{~nm}$ in $6 \mathrm{H}-\mathrm{SiC}$ and continues to increase for other polytypes such as $8 \mathrm{H}$ or $10 \mathrm{H} .{ }^{[2]}$ The cubic polytype on the other hand, due to its symmetry, has equal $\vec{a}$ and $\vec{c}$ lattice constants of $0.435 \mathrm{~nm}$.

The excitonic indirect bandgaps of the polytypes appear to scale between the lowest value, which is $2.3 \mathrm{eV}$ for the cubic $3 \mathrm{C}-\mathrm{SiC}$ and the highest value, which is $3.33 \mathrm{eV}$ for the hexagonal $2 \mathrm{H}-\mathrm{SiC}$, with the other polytypes situ- 
ated in between these two $\left(\mathrm{E}_{g}=3.26 \mathrm{eV}\right.$ for $4 \mathrm{H}-\mathrm{SiC}$ and $\mathrm{E}_{g}=3.02 \mathrm{eV}$ for $6 \mathrm{H}-\mathrm{SiC})$. To understand this, one must realize that, while the cubic polytype $(3 \mathrm{C})$ is $100 \%$ cubic and the hexagonal polytype $(2 \mathrm{H})$ is $100 \%$ hexagonal, all other polytypes are a mixture of the two in terms of stacking sequence. The bandgap has experimentally been found to vary as a function of their degree of hexagonality. ${ }^{[?]}$ As a result of these large bandgaps, several material properties of the commercially most interesting polytype, 4H-SiC, are far superior to $\mathrm{Si}\left(\mathrm{E}_{g}=1.1 \mathrm{eV}\right)$ for use in high-voltage, high-power devices. A good example herefor is the breakdown field strength of $\approx 2.2-2.5 \mathrm{MV} / \mathrm{cm}$ $(4 \mathrm{H}-\mathrm{SiC}$ or $6 \mathrm{H}-\mathrm{SiC})$, which is about ten times larger than that found in $\mathrm{Si}$.

A side effect of the complex stacking sequences of $\mathrm{SiC}$ polytypes, which is relevant for the crystal point defects discussed later, is the existence of inequivalent lattice sites. These sites are best seen in a projection of the atomic positions onto a $(11 \overrightarrow{2} 0)$ plane shown in Fig. 2.2. While the nearest neighbor atomic structure for $\mathrm{Si}$ or $\mathrm{C}$ atoms is always identical in all polytypes, the coordination of the next nearest neighbors may differ in the mixed polytypes. At turning points of the zigzag pattern in the non-cubic polytypes, the local environment of the $\mathrm{Si}-\mathrm{C}$ pair is $2 \mathrm{H}$-like and quasi-hexagonal (denoted $h$ ), whereas between turning points the local environment is 3Clike and quasi-cubic (denoted $k$ ). The total number of inequivalent lattice sites for the mixed polytypes is thus

one quasi-hexagonal $(h)$ and one quasi-cubic $(k)$ site in $4 \mathrm{H}-\mathrm{SiC}$, and

one quasi-hexagonal $(h)$ and two quasi-cubic $\left(k_{1}\right.$ and $\left.k_{2}\right)$ sites in $6 \mathrm{H}-$ $\mathrm{SiC}$.

The purely cubic $3 \mathrm{C}$-SiC of course only has $k$-sites while the purely hexagonal $2 \mathrm{H}$-SiC only has $h$-sites. 
CHAPTER 3

POINT DEFECTS

Crystal defects in general can be categorized according to their dimensionality, from two-dimensional stacking faults over one-dimensional dislocation line defects down to (more or less) zero-dimensional point defects. In $4 \mathrm{H}-$ $\mathrm{SiC}$, the basal plane dislocation, which, under forward bias, may split up into two partials bounding a stacking fault, has received much attention in the past as a device-killing defect. The focus of this thesis, however, lies on point defects, which may come in the form of substitutional (f.e $\mathrm{B}_{C}$ ) or interstitial impurities, vacancies $\left(\mathrm{V}_{S i}\right.$ or $\left.\mathrm{V}_{C}\right)$, self-interstitials $\left(\mathrm{C}_{i}\right.$ or $\left.\mathrm{Si}_{i}\right)$ or antisites $\left(\mathrm{C}_{S i}-\mathrm{Si}_{C}\right)$. Extrinsic foreign atoms substituting for $\mathrm{Si}$ or $\mathrm{C}$ may be useful as dopants, for example $\mathrm{Al}$ or $\mathrm{N}$, or may trigger the formation of (wanted or unwanted) deep compensating centers for the formation of semi-insulating material or also deep recombination centers, as is the case for $\mathrm{V}$ or $\mathrm{Fe}$, respectively. Combinations of basic intrinsic defects are also possible, for example Frenkel pairs $\left(\mathrm{C}_{i}-\mathrm{V}_{C}\right)$, di-vacancies $\left(\mathrm{V}_{C}-\mathrm{V}_{S i}\right)$, split interstitials, where one atom takes the place of two lattice sites, or even larger defect clusters such as carbon interstitial aggregates. Some intrinsic defects may act as recombination centers, such as $\mathrm{V}_{C}$ while others are not electrically active.

Point defects and deep levels in the bandgap associated with them are generally unavoidable in semiconductor crystal growth and device processing. From a thermodynamic viewpoint, a non-zero point defect concentration at thermodynamic equilibrium is an unavoidable result of the minimization of the total free enthalpy of the crystal, determined by the enthalpy and entropy of formation for each specific type of defect. Together with nonequilibrium growth kinetics, which differ between crystal growth processes and reactors, this then determines the final point defect concentration in an as-grown crystal. Later device processing steps, such as implantation, inductively coupled plasma (ICP) etching, thermal oxidation, electron irradiation or electron-beam metallization can lead to lattice damage by introducing foreign atoms and displacing lattice atoms or by selectively destroying the lattice altogether.

In the following sections, first energy levels introduced into the bandgap by defects are discussed, and the differences between shallow and deep levels. Next follows a review of the capture and emission processes of a defect capable of capturing a single charge carrier and the resulting rate equations. 
This is then followed by a discussion of possible capture mechanisms, their temperature dependencies and the emission processes, which are used to model the measurements in this work.

\subsection{Shallow and deep levels in the bandgap}

From a thermodynamical point of view, the bandgap between the conduction $\left(\mathrm{E}_{C}\right)$ and valence $\left(\mathrm{E}_{V}\right)$ bands of a semiconductor can be defined as the sum of the chemical potentials, or the increase of the Gibbs free energy $G$, required for the formation of an electron-hole pair in the crystal. ${ }^{[4]} \mathrm{A}$ point defect locally changes the crystal potential and can lead to the introduction of additional electronic states into the semiconductor, sometimes resonant with either the conduction or the valence band, but often located in the bandgap between them. When an electron is trapped at these point defects, a change in the defect charge state occurs, and the energy levels of a defect can thus be defined as the change of the chemical potential required for the formation of a free carrier and an ionized defect. ${ }^{[4,5]}$

Depending on the absolute charge state of the point defect, these can be divided into donor or acceptor defects. A donor defect is neutral (0) when occupied but attains a positive charge $(+)$ upon ionization, i.e. the release of an electron. An acceptor on the other hand is negatively charged (-) when occupied by an electron and becomes neutral (0) upon electron emission. An alternative description of this process can be made by saying that the acceptor changes from neutral (0) to negative (-) upon emission of a hole.

Another way to distinguish between different types of defects is by separating them into shallow and deep defects. This distinction can be made according to their energy level position inside the bandgap, or, by a more useful distinction, according to the shape of their potential well. A shallow defect can be described as having a widely extended (delocalized) wave function which interacts with only one of the bands and has its ground state close to either the valence or conduction band. Models used to describe these hydrogen-like Coulombic potential wells of shallow defects are, for example, the hydrogenic effective mass theory or the generalized effective mass theory. Due to the shallowness of their energy level, the loosely bound electrons of shallow defects can be ionized at low temperatures and may then move freely through the crystal, similar to an electron in free space albeit with a different effective mass $\mathrm{m}^{*}$. Defects with shallow levels are thus used for doping and conductivity control in semiconductors. Dopants which are generally used in $\mathrm{SiC}$ (where both atoms belong to group IV on the periodic table) are the group III elements aluminum and boron for $p$-type doping and the group $\mathrm{V}$ element nitrogen for $n$-type doping. As is the case for other wide bandgap semiconductors, for example GaN and diamond, $p$-type doping is more difficult than $n$-type doping due to the rather deep energy levels of the $p$-type dopants. For comparison, the thermal activation energy of nitrogen has been found to be $59-102 \mathrm{meV}$, while that of aluminum is 190 $\mathrm{meV}$, i. e. approximately twice as deep inside the bandgap. ${ }^{[6]}$ )

Deep levels on the other hand are best described as possessing short range, steep potential wells, with the electron wave functions spatially localized at the defect site, leading to a delocalization in $\vec{k}$-space. In contrast to shallow levels, the strong binding of an electron to a deep level may result 
in changes in the defect-lattice bonding configuration and lattice vibrations upon emission of the electron. The bonding reconfiguration and the lattice vibrations upon emission lead to an entropy change $(\Delta S)$, which in turn leads to a difference between the Gibbs free energy, or actual energy level $\left(\Delta \mathrm{G}=\mathrm{E}_{C}-\mathrm{E}_{T}\right)$ of the defect and its activation energy or enthalpy $\Delta \mathrm{H}$, given by the thermodynamic relation

$$
\Delta G=\Delta H-T \Delta S
$$

For shallow levels, no bonding reconfiguration occurs after emission of the one loosely bound electron or hole, and $\Delta \mathrm{G}=\Delta \mathrm{H}$.

Deep levels may also capture more than one electron, and in papers 4 and 6 I discuss deep levels capable of capturing and emitting two electrons. In the most common case, the capture of a second charge leads to a weaker binding of the electrons to the defect due to Coulombic repulsion between both the captured charges. In this case, the energy level for the second transition, i.e. capture or emission process, is located closer to the band edge than that of the first transition. This configuration is referred to as a positive- $\mathrm{U}$, due to the positive correlation energy gain upon capture of the second charge. ${ }^{[7]}$ In some cases however, the energy gained by a defect reconfiguration upon capture of a second charge outweighs the Coulombic repulsion between both charges, pushing the second transition deeper into the bandgap and away from its respective band. These are referred to as negative- $\mathrm{U}$ configurations.

\subsection{Capture, emission and rate equations for a single level trap}

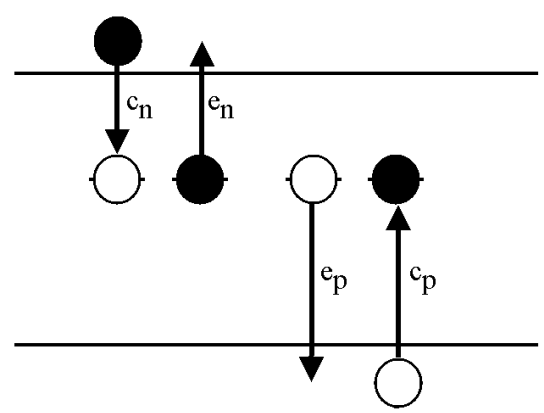

Figure 3.1: The four interaction processes of a single level with the bands electron capture (a), electron emission (b), hole capture (c) and hole emission $(d)$.

A depiction of the hole and electron capture and emission processes possible for a single level defect are given in Fig. 3.1. An electron may be captured from the conduction band (a) with a capture rate $\mathrm{c}_{n}$, after which it can either be re-emitted to the conduction band again with emission rate 
$\mathrm{e}_{n}$ (b) or the electron occupied level can capture a hole with capture rate $c_{p}$ (c). The hole can then be either re-emitted to the valence band at the rate $\mathrm{e}_{p}$ (d) or facilitate the capture of a conduction band electron (a). If a process (a) is followed by a process (c) we refer to this as a recombination event, the inverse of which ((b) followed by (d)) is a generation event. All rates are measured in the unit of charge carriers emitted or captured per second.

The basic rate equations describing the rate of change of excess electrons $n$ or holes $p$ by trapping or recombination at a deep level (DL) can generally be written as

$$
\begin{aligned}
& -\left.\frac{\partial n}{\partial t}\right|_{D L}=(a)-(b)=c_{n} p_{T} n-e_{n} n_{T} \\
& -\left.\frac{\partial p}{\partial t}\right|_{D L}=(c)-(d)=c_{p} p_{T} p-e_{p} p_{T} \\
& \left.\frac{\partial n_{T}}{\partial t}\right|_{D L}=\frac{\partial p}{\partial t}-\frac{\partial n}{\partial t}
\end{aligned}
$$

where $N_{T}, n_{T}$ and $p_{T}=N_{T}-n_{T}$ are the total deep level concentration, and the concentration of electron occupied and of unoccupied deep levels, respectively. Knowledge of the capture rates for electrons $\left(c_{n}\right)$ and holes $\left(c_{p}\right)$ as well as the emission rates for electrons $\left(e_{n}\right)$ and holes $\left(e_{p}\right)$ is thus required to be able to quantify the effect of a deep levels on the charge carriers inside a crystal. Ideally, the capture and emission rates of a deep level are measured independently.

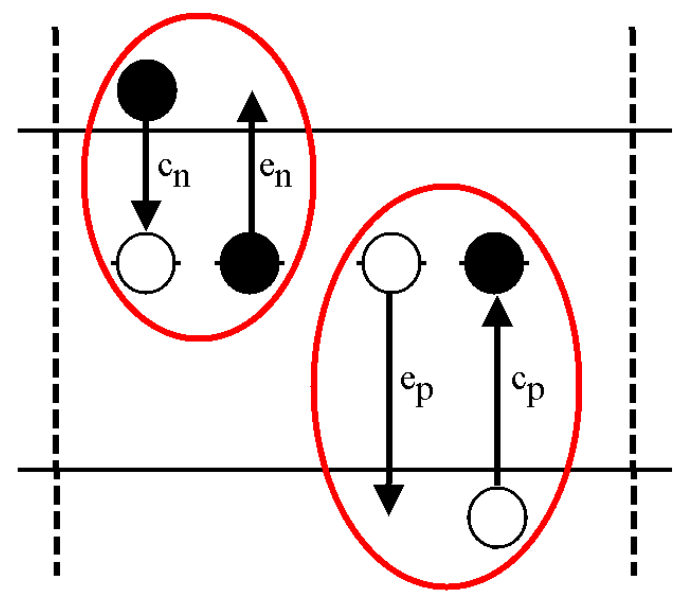

Figure 3.2: In thermal equilibrium, no net flow occurs and the principle of detailed balance requires each process to be counterbalanced by its inverse.

To derive the capture and emission rates, one may invoke the principle of detailed balance, depicted in Fig. 3.2, which states that each rate for a fundamental transition process must be balanced out by its inverse process, independently of other processes occurring around them. The principle of detailed balance is valid in thermal equilibrium as a consequence of another, more widely applicable principle, namely that of microscopic reversibility, 
according to which a transition probability for a process and its inverse must be equal. Examples of such fundamental processes in semiconductors are radiative capture, which in equilibrium must be in balance with its inverse, the rate of optical absorption, or the emission and capture rates of carriers at a deep level.

The principle of detailed balance allows us to relate the emission coefficients to the capture coefficients, or, more precisely, define the ratio between the emission and capture coefficients. This ratio is directly proportional to the concentration of filled and empty deep levels, which depends on the density of charge carriers (holes $\mathrm{p}_{e q}$ or electrons $\mathrm{n}_{e q}$ ) available for capture and must be constant in equilibrium

$$
\begin{aligned}
& \frac{e_{n, e q}}{c_{n, e q}}=\frac{p_{T, e q}}{n_{T, e q}} n_{e q}=n_{1} \\
& \frac{e_{p, e q}}{c_{p, e q}}=\frac{n_{T, e q}}{p_{T, e q}} p_{e q}=p_{1}
\end{aligned}
$$

The subscript $e q$ here denotes equilibrium values.

When dealing with non-equilibrium situations, one generally made assumption is that the emission and capture rates remain more or less equal to their equilibrium values. This assumption is difficult to test but thought to be valid for deviations from the equilibrium which are small and it is also made throughout this thesis.

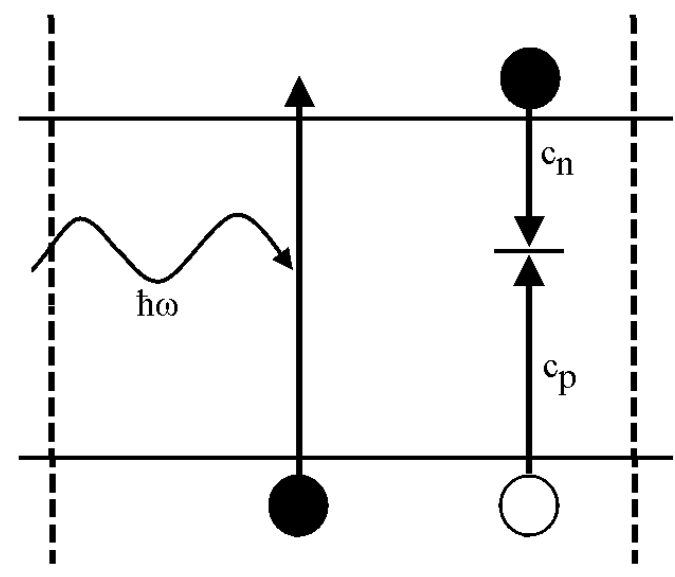

Figure 3.3: In steady state, no net flow occurs but the balance may be held by unrelated processes.

Two other conditions in which a system may be are either transient conditions (Fig. 3.4) or a non-equilibrium steady state (Fig. 3.3). In steady state, no net flow of charge carriers occurs, making this state superficially similar to equilibrium. However, the balance may now be held constant by the interaction of entirely different processes, and not necessarily the balancing out of each fundamental process with its inverse. The often used analytical Shockley-Read-Hall model, which is not used in this thesis, was derived for recombination under steady-state conditions. 


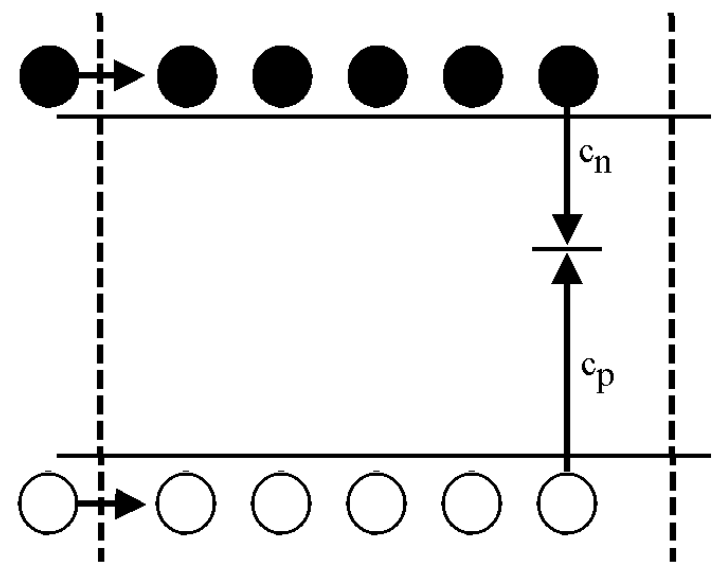

Figure 3.4: Under transient conditions, a net flow occurs as the system returns to its starting point.

Under transient conditions, however, a net flow occurs as the nonequilibrium charge carrier and defect concentrations re-establish their equilibrium (or steady-state) concentrations. Examples for transient conditions, which can be modeled by solution of equations (3.2), (??) and (3.4), are the refilling of traps in DLTS and OE-MCTS filling pulse measurements (papers 4 and 6 ) and the decay of excess carriers monitored by TRPL after an optical injection pulse (modeled numerically in papers 1, 5 and 6 ).

Since the focus of this work is on bulk deep levels and their trapping and recombination processes, spatial net flow through the material is neglected and the focus is only on the movement of charge carriers between conduction and valence band. This is akin to assuming that the concentrations of carriers and defects in equilibrium and non-equilibrium conditions are uniform throughout the material. Recombination and trapping by surface states is also ignored.

\subsection{Capture and recombination processes}

In general, a simple real-space model is used which turns the recombination center into a sphere of a certain radius situated in the crystal lattice. In the cases when the real-space location of a diffusing charge carrier then happens to overlap with the defect sphere, it is captured. In this simple model, the sphere cross sections (denoted as the capture cross sections for electrons $\sigma_{n}$ and holes $\sigma_{p}$ ) and the thermal velocities $\left\langle v_{t h, n / p}\right\rangle$ as well as the densities of available charge carriers and unoccupied defects are the main parameters needed to give the capture rate for electron capture

$$
\begin{aligned}
\left.\frac{\partial n}{\partial t}\right|_{D L} & =-\sigma_{n}\left\langle v_{t h, n}\right\rangle p_{T} n \\
c_{n} & =\sigma_{n}\left\langle v_{t h, n}\right\rangle
\end{aligned}
$$


or for hole capture

$$
\begin{aligned}
\frac{\partial p}{\partial t} \mid & =-\sigma_{p}\left\langle v_{t h, p}\right\rangle n_{T} p \\
c_{p} & =\sigma_{p}\left\langle v_{t h, p}\right\rangle
\end{aligned}
$$

The capture mechanisms for deep levels can be separated into three groups based on their energy dissipation mechanism: Radiative transitions, where the energy is emitted in the form of a photon; phonon-assisted nonradiative transitions, for example multiphonon capture, cascade capture and the two-stage cascade capture model; ${ }^{[8-10]}$ and Auger processes, for example the trap impurity and excitonic Auger capture mechanisms, in which the excess energy is transferred to another charge carrier. ${ }^{[11-13]}$

The radiative mechanism is only of very minor importance in $\mathrm{SiC}$ for recombination above liquid Helium temperatures since $\mathrm{SiC}$ is an indirect bandgap material. It is used the the TRPL simulations, however, to model the TRPL decay curves.

Phonon-assisted non-radiative transitions are of far greater significance in $\mathrm{SiC}$ and will be discussed in more detail. In the multiphonon capture model developed by Henry, et al., a deep level potential which is localized in $\vec{r}$-space but delocalized in $\vec{k}$-space is allowed to interact with phonons of different energies. ${ }^{[8]}$ At elevated temperatures, which must be enough to overcome a capture activation barrier $E_{\infty}$, the lattice vibrations cause a crossing of the deep energy level and the conduction band and enable the capture of the delocalized charge carrier state in one of the bands into the localized defect state. The now filled deep level transitions down to its ground state under emission of multiple phonons. Due to the requirement of phonons for the capture process, the capture probability increases exponentially with temperature (3.11).

$$
\sigma(T)=\sigma_{\infty} \times \exp -\frac{E_{\infty}}{k_{B} T}
$$

The vibronic coupling was found to produce large capture cross sections even for neutral defects, and $\sigma_{\infty}$ was generally found to fall into the range $10^{-14}-10^{-15} \mathrm{~cm}^{2}$.

The cascade capture process is thought to be the main capture mechanism for shallow defects with a hydrogen-like potential, providing a set of closely spaced excited levels with distances less than the maximum phonon energy between each other. ${ }^{[9]} \mathrm{A}$ free charge carrier which diffuses into range of the attractive screened Coulomb potential of the defect can relax to the ground state by dissipating its excess energy in the form of a single phonon at each step. To accomodate this, the steps must have energy separations which are less than the maximum phonon energy. Multiple phonon emissions at the steps are also possible but less likely. Since the levels are closely spaced, the chance for re-emission increase with temperature, giving the capture probability (and thus the capture cross section) a negative power law temperature dependence of the form

$$
\sigma(T)=\sigma_{0} T^{-\alpha}, \quad \alpha=1 \ldots 4
$$

The exact exponent depends on the shape of the defect potential, the energies of the involved acoustical or optical phonons and some assumptions 
in the calculation. As the mechanism requires excited states near one of the bands, it cannot be used to describe capture by non-attractive or repulsive centers. A discussion and modification of the cascade capture model for deeper levels is given by Abakumov and Yassievich, where capture into a deeper defect was shown to be possible by a capture mechanism involving a cascade process as the initial rate limiting step, followed by further steps with different mechanisms. ${ }^{[14]}$ The general idea was also worked out by Gibb, et al., and is described next.

The two-stage cascade capture model of Gibb, (3.13) combines a cascade step into a first shallow excited state of depth $\mathrm{E}_{1}$ close to a band edge, followed by a rapid multiphonon emission process with barrier $\mathrm{E}_{\infty}$ as the charge carrier transitions to the ground state. ${ }^{[10]}$

$$
\sigma(T)=T^{-2} \times \exp \frac{\Delta E}{k_{B} T}, \quad \Delta E=E_{1}-E_{\infty}
$$

The exact form of the temperature dependence, which can be negative or positive and is a mixture of an exponential and a power law term, is thus a function of the energy difference between these two steps. Since the model requires the deep level to have excited states, it can only be used to describe capture into attractive levels. Electron capture into the oxidation induced ON and OS defects (paper 6) as well as into the deep donor levels of the $\mathrm{V}_{C}$-defect, $\mathrm{EH}_{6 / 7}$ (paper 4) appear to proceed via excited states in a fashion described by this model.

The standard or classical Auger process can be used to describe bandto-band non-radiative recombination. Two charge carriers may recombine by transferring their excess energy, which is on the order of the bandgap, to a third particle. The process is thus charge carrier concentration dependent and the rates $\left(R_{n}\right.$ or $\left.R_{p}\right)$ for the three particle process are proportional to the product of the concentrations of the involved carriers, $R_{n}=\gamma_{n} \mathrm{n}^{2} \mathrm{p}$ or $R_{p}=\gamma_{p} \mathrm{p}^{2} \mathrm{n}$, with the Auger coefficients $\gamma_{n, p}$. Due to the concentration dependence, this process is only relevant at very high injection levels which are never reached in the TRPL experiments.

A deep level moderated Auger process, which has been shown to be important in describing recombination processes in $\mathrm{Si}$, is the excitonic Auger mechanism. ${ }^{[12,13]}$ Similar to the classical Auger process, it is only active under bipolar conditions, which occur in TRPL or DLTS measurements when using pn-junctions. In this process, electron-hole pairs form localized free excitons at deep levels, leading to the capture of one particle while the other particle takes over the excess energy. The temperature dependence is mainly a function of the thermal ionization of free excitons, so the excitonic Auger capture cross sections is constant at rougly below liquid nitrogen temperatures but decreases above this temperature

$$
\sigma(T)=A T^{-\alpha}
$$

where $A$ is a proportionality constant and $\alpha$ is the exponent, which is always larger than zero. The process can occur irrespective of the charge state of the deep level since the excitons formed are neutral themselves.

The most important Auger process for this thesis is the trap Auger mechanism or impurity Auger mechanism. ${ }^{[11]}$ In this process, a defect 
must be occupied by two charge carriers (electrons or holes) so that it may capture a charge carrier of opposite type. Upon recombination of an electron and a hole at the defect, the excess energy is transferred to the remaining charge carrier which is then kicked into its respective band. Similar to the classical Auger process, the trap Auger mechanism is only weakly temperature dependent.

As a final word of cauton, despite the large number of possible capture mechanisms and their different temperature dependencies, it is experimentally often difficult to distinguish between specific mechanism, especially when the temperature range is rather narrow, as is the case in DLTS. Even a seemingly clear assignment should thus not be over-interpreted.

\subsection{Emissions from deep levels}

After the preceding discussion of the rate equations governing recombination and trapping via deep levels, the capture rate at deep levels and the possible capture processes which may occur, the emission rate is now discussed. Assuming again that the principle of detailed balance holds, the emission rate of electrons and holes from a level is related to their capture cross section by the following equation

$$
e_{n / p}=\sigma_{n / p}(T)\left\langle v_{t h, n / p}\right\rangle N_{C / V} \frac{g_{0}}{g_{1}} \exp \left(-\frac{\Delta G}{k_{B} T}\right)
$$

where $\frac{g_{0}}{g_{1}}$ is the change in degeneracy of the deep level on emission of the carrier and $\Delta \mathrm{G}$ is the Gibbs free energy discussed in section 3.1 and in eq. (3.1), repeated here for convenience

$$
\Delta G=\Delta H-T \Delta S
$$

We can thus rewrite (3.15) as

$$
\begin{aligned}
e_{n / p} & =\sigma_{n / p}(T) \chi\left\langle v_{t h, n / p}\right\rangle N_{C / V} \exp \left(-\frac{\Delta H}{k_{B} T}\right) \\
\chi & =\frac{g_{0}}{g_{1}} \exp \left(-\frac{\Delta S}{k_{B}}\right)
\end{aligned}
$$

where the entropy and degeneracy factors are summed up in the quantity $\chi$, since they cannot be clearly separated from one another in a DLTS experiment. From this equation it also becomes clear that by fitting only a single DLTS spectrum, we further cannot clearly separate the contributions of the temperature dependent capture cross section $\sigma_{n / p}(T)$ and the enthalpy $\Delta H$ to the emission rate $\mathrm{e}_{n / p}$. This becomes only possible once we analyze several DLTS spectra made using filling pulses of different lengths, which gives us a direct measure of $\sigma_{n / p}(T)$. Traditionally, the analysis is then performed by finding the peak center and creating an Arrhenius plot of the logarithm of the emission rate versus $\frac{1000}{T}$. In papers III, IV, V and VI however I demonstrate that numerically fitting multiple spectra simultaneously with the same parameters, where the peak amplitudes are determined by an appropriate capture model, delivers far superior results.

A number of effects which may distort deep level measurements but which are not discussed here are, for example, deep level or doping profiles within the measured region of the semiconductor, surface recombination 
and trapping, or an enhancement of thermal emission of charge carriers by electric-field induced distortion of the defect potential, resulting in a lowering of the emission barrier (the Poole-Frenkel effect). We refer to the literature for a further description of these effects. ${ }^{[15-17]}$ 


\title{
CHAPTER 4 SCHOTTKY DIODES AND SPACE CHARGE REGIONS
}

\begin{abstract}
Almost all samples used in the capacitance transient experiments are Schottky diodes, where a metal has been deposited onto the semiconductor by thermal or e-beam evaporation. The result is a Schottky barrier which allows the formation of a space charge region, two concepts which are explained in the following for $n$-type material.
\end{abstract}

While an Ohmic contact allows conduction across a metal-semiconductor interface in both directions of bias, forward and reverse, a Schottky barrier can be used to control the direction of charge carrier transport across the interface, allowing conduction to occur when biased in forward direction while blocking transport when biased in reverse. The barrier is the product of the mismatch of the energy levels for majority carriers across the interface of the metal and the semiconductor. A basic theory for the Schottky barrier, which does not include interaction between the metal and the semiconductor at the interface, was developed by W. Schottky and N. Mott. ${ }^{[18,19]}$ In real metal-semiconductor junctions the situation is more complicated. Fermilevel pinning by surface states may for example occur, or the metal and the semiconductor may react at elevated temperatures. Theories accounting for more complex effects such as overlap of the metal and semiconductor wave functions at the interface resulting in charge dipoles have been developed and we refer to the review paper by R. T. Tung for details, while focusing on the simple model below. ${ }^{[20]}$

In the simple model, the Schottky barrier height between a metal with work function $\phi_{M}$ and a semiconductor with electron affinity $\chi_{S}$ is given by

$$
\Phi_{b}=\phi_{M}-\chi_{S}
$$

which results in band bending at the interface as depicted in Fig. 4.1. The band bending then defines a space charge region of extension $W$, from which mobile charge carriers in the semiconductor are expelled, producing a net charge inside the region originating from the ionized, positively charged donor impurities. Deep levels situated inside the space charge region and which are energetically located above the Fermi level $E_{F}$ will now emit their trapped charges by thermal ionization, diminishing the total charge $Q$ stored in the space charge region. The change in capacitance resulting from 


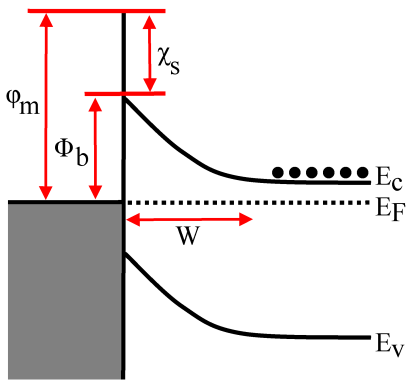

Figure 4.1: The Schottky model of the interface between a metal and an $n$-type semiconductor, where $\mathrm{E}_{C}$ denotes the conduction band edge, $\mathrm{E}_{V}$ the valence band edge, $\mathrm{E}_{F}$ the Fermi level and $W$ the width of the space charge region.

the charge emission at a fixed reverse bias voltage $V$ in the space charge region under a diode of area $A$ is

$$
C=A \frac{d Q}{d V}
$$

takes an exponential or non-exponentially decaying form. It is then recorded as a function of temperature and related to the emission equation (3.17) in the following capacitance transient measurement techniques. 


\section{CHAPTER 5 EXPERIMENTAL METHODS}

The main experimental techniques used in this thesis were capacitance transient methods and time-resolved photoluminescence, which will both be described in the following chapter. Other methods, which have been used in a supporting function to the main methods, are capacitance-voltage profiling of the effective doping concentration and current-voltage measurements of the sample conductivity in forward and reverse. For a detailed description of these supporting methods we refer, however, to textbooks. ${ }^{[15,16]}$

\subsection{Capacitance transient measurements (DLTS, MCTS, O-DLTS, EO-DLTS and OE-MCTS)}

In standard DLTS, the space charge region underneath the Schottky barrier is periodically expanded and collapsed (referred to as the filling pulse). Using n-type material as an example, traps whose energy levels drop below the Fermi level during the filling pulse period may capture electrons for the duration of the filling pulse. Once the space charge region is re-established and the effect of the electric field forces the trap energy levels above the Fermi level again, these traps may emit their electrons. The emission rate is a function of temperature and the emission process results in a temperature dependent capacitance transient.

The measured capacitance transient signal is multiplied by a correlation function $\mathrm{W}(\mathrm{t})$ defined over time interval $\left[\mathrm{t}_{d}\right.$ to $\left.\mathrm{t}_{c}\right]$, which acts as a filter for emissions of its specified emission rate. The product is then averaged over period of correlation $\mathrm{t}_{c}-\mathrm{t}_{d}$.

$$
S\left[t_{c}, t_{d}\right]=\frac{1}{t_{c}} \int_{t_{d}}^{t_{d}+t_{c}} \Delta C(t) W(t) d t
$$

The correlator outputs for different specified emission rates are then plotted as a spectrum over temperature, with peaks occurring at points where emission of the specified emission rate is occurring. These peaks are related to deep levels in the material and their position and shape depends on the trap energy level and capture cross section in eq. (3.17). 
The shape of the weighting function determines its selectivity, or resolution, and its sensitivity to the signal-to-noise ratio. The main advantage of using correlation functions over other methods in the analysis of the transient is their insensitivity to baseline offset, which is a problem for other methods such as direct transient fitting. ${ }^{[21]}$ In case of very low signal levels, a low resolution cosine correlation function is used. [22? ? ] For most work in this thesis, the GS4 function is used. This function was derived based on the Gaver-Stehfest numerical Laplace transform inversion algorithm and offers the best trade-off between SNR sensitivity and selectivity. ${ }^{[23]}$ For high resolution work, the GS6 function offers improved resolution capability but is very sensitive to noise in the transient.

The samples for DLTS were mounted onto aluminum-oxide plates with AgPd contact fingers using Ag-paste as glue. Aluminum bond wires connected the e-beam or thermally evaporated diodes to the contact fingers. The sample was then mounted on a small thermo-chuck, which included a heating cartridge and an RTD temperature detector, and was lowered into a liquid nitrogen bath for cooling, forming a simple dive cryostat. This setup allowed unhindered optical access to the sample and provided temperature stability of up to $\pm 0.1 \mathrm{~K}$. The temperature of the sample was regularly calibrated using a second RTD mounted onto the chuck in a configuration identical to that of a real sample. An Agilent 33220A or Tabor 8024 pulse generator provided electrical pulses down to $50 \cdot 10^{-9} \mathrm{~s}$ length. The central component of the capacitance transient setup was the Boonton 7200 capacitance bridge, which used with an opto-coupler or reed relay controlled pulse switching circuit to bypass the capacitance bridge during application of the electrical filling pulses. For MCTS and O-DLTS, generally the same light source as for the TRPL setup was used together with a software controlled mechanical shutter was used. When optical and electrical pulses were required in a controlled sequence, a second pulse generator running off the same trigger as the shutter was used as a delay generator for the actual pulse generator. The software to control the experiments was written in Labview.

\subsection{Multi-spectrum fitting}

The simplest, and most widely used, parameter extraction method used in DLTS are Arrhenius plots. A Gaussian function is used to fit the top of the DLTS peak and extract the peak center temperature $\left(T_{\text {peak }}\right)$, which is then used in a plot of the emission rates $\ln \left(\frac{T^{2}}{e_{n}}\right)$ versus the inverse of $\frac{1}{T_{p e a k}}$. One may then extract the parameters in eq. (3.17) from the slope and the intercept at $\frac{1000}{K}=0$. Since the Arrhenius plot is constructed from one single spectrum, the parameters cannot be separated well enough, and only an effective activation energy $\mathrm{E}_{n a}$ and an effective capture cross section $\sigma_{n a}$ are extracted. The activation energy is proportional to $\Delta \mathrm{H}$ and includes the temperature dependent parts of the capture cross section model, while $\sigma_{n a}$ is proportional to the capture cross section pre-factor mixed with the entropy and degeneracy terms. The method, however, generally fails or delivers erroneous results when peaks overlap in the spectrum, since the Gaussian function is not connected to any physical model and thus cannot properly separate overlapping emission processes. The traditionally used correlation functions, such as sine, cosine or rectangular lock-in functions, also provide poor resolution, making peak separation all the more difficult. 
To overcome these problems, Laplace-DLTS can be used, in which a numerical inverse Laplace transform is taken in order to separate different emission rates. The method provides resolution far superior to what is possible with standard DLTS. ${ }^{[24]}$

Another way of improving over standard DLTS, which I have used in this thesis, is multi-spectrum fitting using the higher resolution correlation functions GS4 and GS6. Here, several measurements made with different filling pulse lengths are fitted together using the same set of parameters to numerically simulate the entire DLTS spectrum (eq. (3.17)). The peak heights are determined directly from the temperature dependent capture cross sections (eq. (3.11), (3.12), (3.13), (3.14)) and the filling pulse length. A numerical calculation of slow capture and re-emission in the transition region of the space charge region is also included in the model, which allows to extend the range of filling pulse lengths which can be used for the technique. The software was written in Python.

Single and two-level defects were fitted by this method. The first simulations of two-level defects were made using a model developed by Ferrari et al.. [?]) Later measurements on $\mathrm{EH}_{6 / 7}$ and the oxidation induced defects required expanded models, derived either to allow simultaneous hole and electron capture into both defect levels (paper VI) or hole capture by the trap Auger mechanism to simulate $\mathrm{EH}_{6 / 7}$ (paper IV).

\subsection{Time resolved photoluminescence (TRPL)}

In TRPL, the near band edge luminescence decay, which results from direct band to band radiative electron - hole pair recombination after a short optical injection pulse, is monitored. The intensity of the PL signal is directly proportional to the excess carrier density $(\partial \mathrm{n}(\mathrm{t}), \partial \mathrm{p}(\mathrm{t}))$

$$
I_{P L}(t)=\gamma\left(n_{e q}+\partial n(t)\right)\left(p_{e q}+\partial p(t)\right)
$$

The measurements are often made under low injection conditions, meaning the excess carrier density has dropped below the doping density. This allows us to neglect the term $\partial \mathrm{n}(\mathrm{t})$, and the monitored intensity becomes directly proportional to the decay of the excess minority carrier density $\partial \mathrm{p}(\mathrm{t})$.

Once the decay curve has been recorded, a decay constant is extracted from it. This measured lifetime $\frac{1}{\tau_{\text {measured }}}$ results of the sum of all processes in the semiconductor which recombine the excess injected carriers and return the system to its initial state. It can be written as

$$
\frac{1}{\tau_{\text {measured }}}=\frac{1}{\tau_{N R B}}+\frac{1}{\tau_{S R}}+\frac{1}{\tau_{\text {Auger }}}+\frac{1}{\tau_{\text {Rad }}}
$$

where $\frac{1}{\tau_{N R B}}$ is the non-radiative bulk lifetime, $\frac{1}{\tau_{S R}}$ results from surface recombination, $\frac{1}{\tau_{\text {Auger }}}$ is the contribution from Auger processes and $\frac{1}{\tau_{\text {Rad }}}$ is due to radiative recombination. Since $4 \mathrm{H}-\mathrm{SiC}$ is an indirect bandgap material, radiative processes do little to limit the excess carrier lifetime. Measurements are generally also done under injection levels low enough to make Auger processes irrelevant. This leaves the non-radiative bulk and surface recombination pathways as the main determining factors for the measured 
carrier lifetime.

To extract the decay constants, I either use single exponential fitting to the transient or, in cases when the transient changes shape significantly over the course of the decay, the transient is fitted by a spline function. The first derivative of this spline function is used to calculate the instantaneous lifetime of the curve

$$
\tau_{\text {measured }}(t)=\frac{1}{\left(-\frac{d I_{P L}}{d t}\right)}
$$

In the TRPL setup, the excitation source was a $355 \mathrm{~nm}, 10 \mathrm{kHz}$ Nd:YAG passively Q-switched laser, which used a very fast photodiode to collect a fraction of the laser pulse and convert it into a post-pulse trigger signal for the Agilent U1082A digitizer/digital oscilloscope. In the room temperature TRPL mapping setup, samples were placed on an xy-stage with $150 \mathrm{~mm} x$ $150 \mathrm{~mm}$ travel with $2 \mu \mathrm{m}$ positioning repeatability. The laser focused onto the sample by a lens at a $72 \mathrm{deg}$ angle to the sample surface. A Nikon 20x microscope objective with NA 0.75 collected, parallelized and directed the photoluminescence into a Hamamatsu PMT. A narrow bandpass filter with an appropriate center wavelength was placed in between the objective and the PMT to select out the near band-edge luminescence. The setup for temperature dependent TRPL replaced the short range microscope objective with a long working range Mitutoyo $10 \mathrm{x}$ objective, which only had a NA of 0.42 . The increased distance to the sample was necessary to protect the objective from heat or cold damage by a sapphire window and a cooling fan. The sample holder itself was constructed mainly of high temperature steel and quartz glass. Inside, the sample was glued, using high-temperature silver paste, onto a silicon nitride ceramic heating cartridge manufactured by Bach RC, which heated the sample to a maximum temperature of 1000 $\mathrm{K}$. Liquid nitrogen allowed cooling of the sample giving a total temperature range of $77 \mathrm{~K}-1000 \mathrm{~K}$. Due to an increased background light level by thermal radiation, the transients were averaged for up to 30 minutes at high temperatures, ultimately giving measurements with a dynamic range of at least 4 orders of magnitude. 


\section{CHAPTER 6 SUMMARY OF THE PAPERS}

Paper I A new lifetime limiting defect in $4 \mathrm{H}-\mathrm{SiC}$ grown by chlorinated chemistry, referred to as RB1 is characterized by DLTS, $\mathrm{p} n$-junction DLTS and TRPL. The defect, which occurs in concentrations up to $2 \cdot 10^{14} \mathrm{~cm}^{-3}$ is capable of controlling TRPL measured carrier lifetimes when $\mathrm{Z}_{1 / 2}$ concentrations are low. The thermal activation energy $E_{a}$ and effective electron capture cross section $\sigma(T)$ are determined as $1.05 \mathrm{eV}$ and $2 \cdot 10^{-16} \mathrm{~cm}^{2}$, respectively. From measurements of the TRPL carrier lifetime versus the defect concentration, we propose an effective hole capture cross section of $(5 \pm 2) \cdot 10^{-15} \mathrm{~cm}^{2}$. The defect is linked to an iron contamination resulting from the corrosion of steel reactor parts and the RB1 and Fe1 (a DLTS level related to iron) deep levels are interrelated in a sub-linear way with each other.

Paper II The electron and hole capture rates of all deep levels accessible in $p$-type $4 \mathrm{H}$-SiC by DLTS and MCTS are investigated for their efficiency as recombination centers. The deep levels detected in the top half of the bandgap, LB1 and EM1 are found to be pure electron traps, while the deep levels found in the lower half of the bandgap (HK0, HK2 and the D-center) are all found to be hole traps. HK3 and HK4, which are prominent deep levels in the lower half of the bandgap in epilayers grown at Kyoto University, are not detected in our samples. $\mathrm{EH}_{6 / 7}$, ON1 and ON2, found in the top half of the bandgap and separately characterized in papers IV and VI, were found to be poor recombination centers. We thus conclude that the main lifetime limiting deep levels in $p$-type $4 \mathrm{H}$-SiC must be in the top half of the bandgap and have a capture cross section ratio of $\sigma_{e} \ll \sigma_{h}$, making them undetectable by MCTS. The $\mathrm{Z}_{1 / 2}$ level, which, together with $\mathrm{EH}_{6 / 7}$, is related to the carbon vacancy $\left(\mathrm{V}_{C}\right)$ defect, is determine to be the most likely candidate.

Paper III Carrier lifetimes are measured by TRPL in $p$ - and $n$-type $4 \mathrm{H}-\mathrm{SiC}$ over a wide temperature range $(77-1000 \mathrm{~K})$ and compared. We show that the temperature dependence of the lifetime is identical in both conductivity types. Together with paper II, this is interpreted as a further indication that the carrier lifetime is limited by the same defect, namely $\mathrm{Z}_{1 / 2}$.

Paper IV The near midgap deep level $\mathrm{EH}_{6 / 7}$, which occurs in both 
$n$ - and $p$-type $4 \mathrm{H}-\mathrm{SiC}$ is characterized by high-resolution numerical multi-spectra fitting of DLTS and MCTS measurements measured with different filling pulse lengths. In literature, EH7 has been clearly assigned to a donor transition of the $\mathrm{V}_{C}$-defect (specifically $\mathrm{V}_{C}$ on a hexagonal lattice site), which also produces the two overlapping negative- $\mathrm{U}$ double acceptor-type transitions $\mathrm{Z}_{1 / 2}$. The location of the other donor-type transitions and the assignment of EH6 to a defect has been controversial. By applying multi-level models to the $\mathrm{EH}_{6 / 7}$ peak we show that the peak is actually composed of two negative- $U$ centers, giving rise to four strongly overlapping donor-type transitions. We thus assign $\mathrm{EH}_{6}$ and $\mathrm{EH}_{7}$ to the carbon vacancy.

Paper V Shallow boron and the related deep D-center are shown to produce minority carrier trapping effects in heavily boron co-doped $n$ type $4 \mathrm{H}-\mathrm{SiC}$ epilayers. We simulate TRPL decay curves based on level parameters extracted by MCTS from the $n$-type epilayers and find that this trapping effect may be misinterpreted in room temperature TRPL measurements as a high free carrier lifetime. We further show that the D-center is composed of two strongly overlapping peaks and we characterize the capture behavior of both by filling pulse DLTS made in $p$-type epilayers.

Paper VI Deep levels produced as a by-product of lifetime enhancing oxidation located in the upper half of the $n$ - and $p$-type $4 \mathrm{H}$ - and $6 \mathrm{H}$ $\mathrm{SiC}$ bandgaps, referred to as ON- and OS-defects were characterized by various capacitance transient techniques. The deep levels all appear to be of double donor-type, possessing three charge states $(++,+$ and $0)$. The two electron transition processes of two defects in $4 \mathrm{H}-\mathrm{SiC}$ (ON1 and ON2) give rise to three DLTS peaks, while the three OS1, OS2 and OS3 defects in 6H-SiC produce six DLTS peaks with strong overlap. Numerical multi-spectra fitting of DLTS spectra measured with varying filling pulses and the application of multi-level defect models allowed determining enthalpies $(\Delta H)$, entropy factors $(\chi)$ and the temperature dependence of the capture cross sections $(\sigma(T))$. The electron capture is suggested to occur via excited Coulomb-attractive states near the conduction band edge and is well described by a twostage cascade capture model. Optical-electrical (OE-MCTS) filling pulse measurements in $p$-type showed the defects were only capable of hole capture at a low rate when neutral, making the ON- and OSdefects extremely inefficient recombination centers. 
[1] W. F. Knippenberg. Growth phenomena in silicon carbide. Phillips Res. Report, 18:161-274, 1963.

[2] C.M. Zetterling. Process technology for silicon carbide devices. EMIS processing series no. 2, INSPEC IEE, UK, 2002.

[3] H. Iwata. Stacking faults in silicon carbide. PhD thesis, Linköping university, 2003.

[4] C. D. Thurmond. The standard thermodynamic functions for the formation of electrons and holes in ge, si, gaas and gap. J. Electrochem. Society, 122(8):1133-1141, 1975.

[5] J. A. van Vechten and C. D. Thurmond. Entropy of ionization and temperature variation of the ionization levels of defects in semiconductors. Phys. Rev. B, 14(8):3539-3550, 1976.

[6] W. J. Choyke, H. Matsunami, and G. Pensl, editors. Silicon carbide: A review of fundamental questions and applications to current device technology. Akademie Verlag Berlin, 1. edition, 1997.

[7] P. W. Anderson. Model for the electronic structure of amorphous semiconductors. Phys. Rev. Lett., 34:953, 1975.

[8] C. H. Henry and D.V. Lang. Nonradiative capture and recombination by multiphonon emission in GaAs and GaP. Phys. Rev. B, 15(2):9891016, 1977.

[9] Melvin Lax. Cascade capture of electrons in solids. Phys. Rev., 119: 1502-1523, Sep 1960.

[10] R. M. Gibb, G. J. Rees, B. W. Thomas, B. L. H. Wilson, B. Hamilton, D. R. Wight, and N. F. Mott. V. a two stage model for deep level capture. Philos. Mag., 36(4):1021-1034, 1977.

[11] M. Kleverman, A. Litwin, E. Janzén, , and H. G. Grimmeiss. Capture processes as double donors in si. Phys. Rev. B, 31(6):3659-3666, 1985.

[12] A. Hangleiter. Nonradiative recombination via deep impurity levels in silicon: Experiment. Phys. Rev. B, 35:9149-9161, Jun 1987. 
[13] A. Hangleiter. Nonradiative recombination via deep impurity levels in semiconductors: The excitonic auger mechanism. Phys. Rev. B, 37: 2594-2604, Feb 1988.

[14] V. N. Abakumov, V. I. Perel, and I. N. Yassievich. Nonradiative Recombination in Semiconductors (Modern Problems in Condensed Matter Sciences vol. 33). (Amsterdam: North-Holland), 1991.

[15] Dieter K. Schroder. Semiconductor Material and Device Characterization. John Wiley \& Sons, Inc., third edition edition, 2006.

[16] P. Blood and Orton W. The electrical characterization of semiconductors: Majority carriers and electron states. Academic Press Limited, London, 1992.

[17] S. Rein. Lifetime Spectroscopy: A Method of Defect Characterization in Silicon for Photovoltaic Applications, volume 85 of Springer Series in Material Science. Springer Berlin Heidelberg New York, 2005.

[18] W. Schottky. Z. Physik, 113:367, 1939.

[19] N. F. Mott. Proc. Roy. Soc. (London), 171:27, 1939.

[20] R. T. Tung. Recent advances in schottky barrier concepts. Mater. Sci. Eng. R-Rep., 35:1-136, 2001.

[21] A. A. Istratov and O. F. Vyvenko. Exponential analysis in physical phenomena. Rev. Sci. Instr., 70(2):1233-1257, 1999.

[22] A. A. Istratov, O. F. Vyvenko, H. Hieslmair, and E. R. Weber. Critical analysis of weighting functions for the deep level transient spectroscopy of semiconductors. Meas. Sci. Technol., 9:477-484, 1998.

[23] A. A. Istratov. The resolution limit of traditional correlation functions for deep level transient spectroscopy. Rev. Sci. Instr., 68(10):38613865, 1997.

[24] L. Dobaczewski, A. R. Peaker, and K. Bonde Nielsen. Laplacetransform deep-level spectroscopy: The technique and its applications to the study of point defects in semiconductors. J. Appl. Phys., 96(9): 4689-4728, 2004. 


\section{Papers}

The articles associated with this thesis have been removed for copyright reasons. For more details about these see:

http://urn.kb.se/resolve?urn=urn:nbn:se:liu:diva-121515 